\title{
DERIVATIVES OF INNER FUNCTIONS IN BERGMAN SPACES INDUCED BY DOUBLING WEIGHTS
}

\author{
Fernando Pérez-González, Jouni Rättyä and Atte Reijonen \\ Universidad de La Laguna, Departamento de Análisis Matemático \\ P.O. Box, 456, 38200 La Laguna, Tenerife, Spain; fernando.perez.gonzalez@ull.es \\ University of Eastern Finland, Department of Physics and Mathematics \\ P.O. Box 111, 80101 Joensuu, Finland; jouni.rattya@uef.fi \\ University of Eastern Finland, Department of Physics and Mathematics \\ P.O. Box 111, 80101 Joensuu, Finland; atte.reijonen@uef.fi
}

\begin{abstract}
We find a condition for the zeros of a Blaschke product $B$ which guarantees that $B^{\prime}$ belongs to the Bergman space $A_{\omega}^{p}$ induced by a doubling weight $\omega$, and show that this condition is also necessary if the zero-sequence of $B$ is a finite union of separated sequences. We also give a general necessary condition for the zeros when $B^{\prime} \in A_{\omega}^{p}$, and offer a characterization of when the derivative of a purely atomic singular inner function belongs to $A_{\omega}^{p}$.
\end{abstract}

\section{Introduction and main results}

Let $\mathcal{H}(\mathbf{D})$ denote the space of analytic functions in the unit disc $\mathbf{D}=\{z \in$ $\mathbf{C}:|z|<1\}$ of the complex plane $\mathbf{C}$. A function $\omega: \mathbf{D} \rightarrow[0, \infty)$, integrable over $\mathbf{D}$, is called a weight. It is radial if $\omega(z)=\omega(|z|)$ for all $z \in \mathbf{D}$. For $0<p<\infty$ and a weight $\omega$, the weighted Bergman space $A_{\omega}^{p}$ consists of $f \in \mathcal{H}(\mathbf{D})$ such that

$$
\|f\|_{A_{\omega}^{p}}^{p}=\int_{\mathbf{D}}|f(z)|^{p} \omega(z) d A(z)<\infty
$$

where $d A(z)=\frac{d x d y}{\pi}$ is the normalized Lebesgue area measure on $\mathbf{D}$. As usual, $A_{\alpha}^{p}$ stands for the classical weighted Bergman space induced by the standard radial weight $\omega(z)=\left(1-|z|^{2}\right)^{\alpha}$, where $-1<\alpha<\infty$. For $f \in \mathcal{H}(\mathbf{D})$ and $0<r<1$, set

$$
M_{p}(r, f)=\left(\frac{1}{2 \pi} \int_{0}^{2 \pi}\left|f\left(r e^{i t}\right)\right|^{p} d t\right)^{1 / p}, \quad 0<p<\infty
$$

and $M_{\infty}(r, f)=\max _{|z|=r}|f(z)|$. For $0<p \leq \infty$, the Hardy space $H^{p}$ consists of $f \in \mathcal{H}(\mathbf{D})$ such that $\|f\|_{H^{p}}=\sup _{0<r<1} M_{p}(r, f)<\infty$.

A function $\Theta \in H^{\infty}$ is an inner function if it has unimodular radial limits almost everywhere on the boundary $\mathbf{T}$ of the unit disc $\mathbf{D}$. The question of when the derivative of an inner function belongs to the Hardy or the Bergman spaces has been a subject of research since 1970's. Membership of the derivative in the Hardy space $H^{p}$ and its Banach envelope $B^{p}$, with $0<p<1$, was studied in $[1,3,4,7,20,33]$. Derivatives of inner functions in the weighted Bergman space $A_{\alpha}^{p}$ has been studied in $[2,19,21]$, see

https://doi.org/10.5186/aasfm.2017.4248

2010 Mathematics Subject Classification: Primary 30J10, 30J15; Secondary 30H20.

Key words: Bergman space, Blaschke product, doubling weight, inner function.

This research was supported in part by Ministerio de Economía y Competitivivad, Spain, project MTM2014-52865-P and project MTM2015-69323-REDT; by Academy of Finland project no. 268009; by North Karelia Regional fund of Finnish Cultural Foundation; by Väisälä Fund of Finnish Academy of Science and Letters, and by Faculty of Science and Forestry of University of Eastern Finland project no. 930349. 
$[11,13,14,15,16,17,22,32,34]$ for recent developments. See also the monographs [9] and [25]. Many known results on the classical weighted Bergman space $A_{\alpha}^{p}$ were recently generalized in [6] to the setting of $A_{\omega}^{p}$ induced by a normal weight $\omega$. Recall that a radial weight $\omega$ is called normal if there exist real numbers $a$ and $b$ and $r_{0} \in(0,1)$ such that

$$
\frac{\omega(r)}{(1-r)^{a}} \nearrow \infty, \quad \frac{\omega(r)}{(1-r)^{b}} \searrow 0
$$

for $r>r_{0}$ [35]. Normal weights are essentially constant in hyperbolically bounded sets [6, Lemma 1], hence they cannot oscillate too much, and in particular they do not have zeros. The purpose of this note is to continue the line of investigation of [6] with the difference that we consider weights $\omega$ that are less regular. The class $\widehat{\mathcal{D}}$ of radial weights $\omega$ such that $\widehat{\omega}(z)=\int_{|z|}^{1} \omega(s) d s$ admits the doubling property $\widehat{\omega}(z) \leq C \widehat{\omega}\left(\frac{1+|z|}{2}\right)$ gives a sufficiently general setting for our purposes. Since the definition of $\widehat{\mathcal{D}}$ depends on integrals, it does not require any local smoothness for $\omega$. The point of departure of this study is the recent operator theoretic result which tells, in particular, when the Schwarz-Pick lemma may be applied to the derivative of an inner function in the norm of the Bergman space $A_{\omega}^{p}$ without causing any essential loss of information. More precisely, if $0<p<\infty$ and $\omega \in \widehat{\mathcal{D}}$, then by the main result in [31] the asymptotic equality

$$
\left\|\Theta^{\prime}\right\|_{A_{\omega}^{p}}^{p} \asymp \int_{\mathbf{D}}\left(\frac{1-|\Theta(z)|^{2}}{1-|z|^{2}}\right)^{p} \omega(z) d A(z)
$$

is valid for all inner functions $\Theta$ if and only if

$$
\sup _{0<r<1} \frac{(1-r)^{p}}{\widehat{\omega}(r)} \int_{0}^{r} \frac{\omega(s)}{(1-s)^{p}} d s<\infty .
$$

Writing $\omega \in \widehat{\mathcal{D}}_{p}$ if the supremum above is finite, an immediate consequence of this result is that each subproduct of a Blaschke product $B$ such that $B^{\prime} \in A_{\omega}^{p}$ with $\omega \in \widehat{\mathcal{D}}_{p}$ also has its derivative in $A_{\omega}^{p}$. We also deduce that, for $\omega \in \widehat{\mathcal{D}}_{p}$, the derivative of a finite product $\prod_{j=1}^{n} \Theta_{j}$ of inner functions belongs to $A_{\omega}^{p}$ if and only if $\Theta_{j}^{\prime} \in A_{\omega}^{p}$ for all $j=1, \ldots, n$. Therefore in this case we may consider different types of inner functions separately. Before proceeding further, more definitions on weights are in order. We say that $\omega \in \mathcal{D}$ if there exist $C=C(\omega) \geq 1, \alpha=\alpha(\omega)>0$ and $\beta=\beta(\omega) \geq \alpha$ such that

$$
C^{-1}\left(\frac{1-r}{1-t}\right)^{\alpha} \widehat{\omega}(t) \leq \widehat{\omega}(r) \leq C\left(\frac{1-r}{1-t}\right)^{\beta} \widehat{\omega}(t), \quad 0 \leq r \leq t<1 .
$$

It is known that the existence of $\beta$ such that the right-hand inequality is satisfied is equivalent to $\omega \in \widehat{\mathcal{D}}$ by $[29$, Lemma 1$]$, and therefore $\widehat{\mathcal{D}}=\cup_{p>0} \widehat{\mathcal{D}}_{p}$. It is easy to see that the left-hand inequality is equivalent to the existence of $K=K(\omega)>1$ and $C=C(\omega)>1$ such that the doubling property $\widehat{\omega}(r) \geq C \widehat{\omega}\left(1-\frac{1-r}{K}\right)$ is satisfied for all $0 \leq r<1$. For details and more, see [30].

For a given sequence $\left\{z_{n}\right\}$ in $\mathbf{D}$ for which $\sum_{n}\left(1-\left|z_{n}\right|\right)$ converges, the Blaschke product associated with the sequence $\left\{z_{n}\right\}$ is defined as

$$
B(z)=\prod_{n} \frac{\left|z_{n}\right|}{z_{n}} \frac{z_{n}-z}{1-\bar{z}_{n} z}
$$


A sequence $\left\{z_{n}\right\}$ in $\mathbf{D}$ is called separated (or uniformly discrete) if there exists $\delta>0$ such that

$$
\inf _{k \neq n}\left|\frac{z_{k}-z_{n}}{1-\bar{z}_{k} z_{n}}\right|=\delta
$$

Writing $\omega \in \mathcal{J}_{p}$ if

$$
\sup _{0<r<1} \frac{(1-r)^{p}}{\widehat{\omega}(r)} \int_{r}^{1} \frac{\omega(s)}{(1-s)^{p}} d s<\infty,
$$

the main result of this study on Blaschke products reads as follows.

Theorem 1. Let $\frac{1}{2}<p<\infty$ and $\omega \in \widehat{\mathcal{D}}_{p} \cap \mathcal{D}$. Let $B$ be the Blaschke product associated with a finite union of separated sequences $\left\{z_{n}\right\}_{n=1}^{\infty}$. If either $\frac{1}{2}<p \leq 1$ and $\omega \in \widehat{\mathcal{D}}_{2 p-1}$, or $1<p<\infty$ and $\omega \in \mathcal{J}_{p-1}$, then

$$
\left\|B^{\prime}\right\|_{A_{\omega}^{p}}^{p} \asymp \sum_{n=1}^{\infty} \frac{\widehat{\omega}\left(z_{n}\right)}{\left(1-\left|z_{n}\right|\right)^{p-1}} .
$$

To give some insight to the hypotheses let us take a look at the case $1<p<\infty$ in which $\omega \in \widehat{\mathcal{D}}_{p} \cap \mathcal{J}_{p-1}$. Roughly speaking the containment in $\widehat{\mathcal{D}}_{p}$ says that the integral $\int_{0}^{r} \omega(s) /(1-s)^{p} d s$ must grow as a negative power of $1-r$, and $\omega \in \mathcal{J}_{p-1}$ if $\int_{r}^{1} \omega(s) /(1-s)^{p-1} d s$ tends to 0 as a positive power of $1-r$. In the case of the standard weight $\omega(z)=\left(1-|z|^{2}\right)^{\alpha}$, the requirement $\omega \in \widehat{\mathcal{D}}_{p} \cap \mathcal{J}_{p-1}$ reduces to the chain of inequalities $p-2<\alpha<p-1$. For this special case the result is well known, and is generalized in [6, Theorem 2] for an appropriate subclass of normal weights. Theorem 1 in turn generalizes the last-mentioned result.

In Section 2 we first establish sharp upper bounds for $\left\|B^{\prime}\right\|_{A_{\omega}^{p}}$, when $B$ is a general Blaschke product, by using standard techniques. To show that $A_{\omega}^{p}$-norm of $B^{\prime}$ dominates the sum in (1.3) is more involved and the true difficulty in proving Theorem 1 stems from the fact that $\omega \in \mathcal{D}$ does not admit any local smoothness. We circumvent the problem by using maximal functions, their boundedness and Carleson measures for $A_{\omega}^{p}$. Therefore our reasoning is substantially different from that of $[6$, Theorem 2]. The proof of Theorem 1 is presented in Section 2 where we also point out that the hypothesis $\omega \in \mathcal{D}$ can be relaxed to $\omega \in \widehat{\mathcal{D}}$ if $1 \leq p<\infty$ and $B$ is a Carleson-Newman Blaschke product. The significant difference between the classes $\mathcal{D}$ and $\widehat{\mathcal{D}}$ is that $\widehat{\mathcal{D}}$ contains the so-called rapidly increasing weights that induce Bergman spaces $A_{\omega}^{p}$ lying in a sense much closer to the Hardy spaces $H^{p}$ than any of the standard weighted Bergman spaces $A_{\alpha}^{p}$ [28]. The canonical example of a smooth weight in $\widehat{\mathcal{D}} \backslash \mathcal{D}$ is $v_{\alpha}(z)=(1-|z|)^{-1}\left(\log \frac{e}{1-|z|}\right)^{-\alpha}$ for each $1<\alpha<\infty$.

It is natural to search for necessary conditions for the zeros $\left\{z_{n}\right\}$ of a Blaschke product $B$ when its derivative belongs to $A_{\omega}^{p}$. It is known by [3] that $\sum_{n}\left(1-\left|z_{n}\right|\right)^{\beta}<$ $\infty$ for all $\beta>(1+\alpha) /-\alpha$ if $B^{\prime} \in A_{\alpha}^{1}$ with $-1<\alpha<-1 / 2$. This result was recently generalized in [32] to other values of $p<1$ : If $B^{\prime} \in A_{\alpha}^{p}$, where $3 / 2+\alpha<p \leq 1$, then $\sum_{n}\left(1-\left|z_{n}\right|\right)^{\beta}<\infty$ for all $\beta>(2+\alpha-p) /(p-\alpha-1)$. The case (a) of the next result gives an analogue of these results for $A_{\omega}^{p}$.

Theorem 2. Let $\omega$ be a radial weight, and let $B$ be the Blaschke product associated with a sequence $\left\{z_{n}\right\}_{n=1}^{\infty}$. 
(a) Let $\frac{1}{2}<p \leq 1$. If there exist $\varepsilon>0$ and a constant $C=C(p, \varepsilon, \omega)>0$ such that

$$
\widehat{\omega}(r) \leq C\left(\frac{1-r}{1-t}\right)^{p-\varepsilon} \widehat{\omega}(t), \quad 0 \leq r \leq t<1,
$$

then $\left\|B^{\prime}\right\|_{A_{\omega}^{p}} \gtrsim \sum_{n=1}^{\infty} \widehat{\omega}\left(z_{n}\right)^{\frac{1}{\varepsilon}}\left(1-\left|z_{n}\right|\right)^{\gamma}$ for all $\gamma>\frac{1-p}{\varepsilon}$.

(b) Let $1<p<\infty$. If there exist $\varepsilon>p-1, \frac{1-p}{1+\varepsilon-p}<\gamma<0$ and a constant $C=C(p, \varepsilon, \omega, \gamma) \geq 1$ such that

$$
C^{-1}\left(\frac{1-r}{1-t}\right)^{\gamma(p-\varepsilon-1)} \widehat{\omega}(t) \leq \widehat{\omega}(r) \leq C\left(\frac{1-r}{1-t}\right)^{p-\varepsilon} \widehat{\omega}(t), \quad 0 \leq r \leq t<1,
$$

then $\left\|B^{\prime}\right\|_{A_{\omega}^{p}}^{p} \gtrsim \sum_{n=1}^{\infty} \widehat{\omega}\left(z_{n}\right)^{\frac{1}{1+\varepsilon-p}}\left(1-\left|z_{n}\right|\right)^{\gamma}$.

If $\omega(z)=(1-|z|)^{\alpha}$, then $\widehat{\omega}\left(z_{n}\right)^{\frac{1}{\varepsilon}}\left(1-\left|z_{n}\right|\right)^{\gamma} \asymp\left(1-\left|z_{n}\right|\right)^{\frac{\alpha+1}{\varepsilon}+\gamma}$. Since $\gamma>(1-p) / \varepsilon$, we have $(\alpha+1) / \varepsilon+\gamma>(2+\alpha-p) / \varepsilon$, where $\varepsilon \leq p-\alpha-1$ by the hypothesis (1.4). Thus [32, Theorem 1] follows from Theorem 2. Further, (b) shows that if $B^{\prime} \in A_{\alpha}^{p}$ with $\alpha<0$ and $p>\max \{1,2(1+\alpha)\}$, then $\sum_{n}\left(1-\left|z_{n}\right|\right)^{\beta}<\infty$ for all $\beta>(p-2) / \alpha-1$. This is a natural counterpart of [3, Theorem 6] for $p>1$.

The proof of Theorem 2 is given at the end of Section 2. The argument we employ uses ideas from the proofs of [3, Theorem 6] and [32, Theorem 1]. The presence of a general weight $\omega$ instead of the standard weight causes technical obstructions in the argument, but also allows us to make certain parts of the proof more simple and transparent. Therefore Theorem 2 can be considered as a streamlined generalization of $[3$, Theorem 6$]$ and [32, Theorem 1].

Singular inner functions are of the form

$$
S_{\sigma}(z)=\exp \left(\int_{\mathbf{T}} \frac{z+w}{z-w} d \sigma(w)\right), \quad z \in \mathbf{D}
$$

where $\sigma$ is a positive measure on $\mathbf{T}$, singular with respect to the Lebesgue measure. If the measure $\sigma$ is purely atomic, then this definition reduces to the form

$$
S(z)=\prod_{n} \exp \left(\gamma_{n} \frac{z+\xi_{n}}{z-\xi_{n}}\right)=\exp \left(\sum_{n} \gamma_{n} \frac{z+\xi_{n}}{z-\xi_{n}}\right), \quad z \in \mathbf{D},
$$

where $\xi_{n} \in \mathbf{T}$ are distinct points and $\gamma_{n}>0$ satisfy $\sum_{n} \gamma_{n}<\infty$. This type of functions are known as purely atomic singular inner functions associated with $\left\{\xi_{n}\right\}$ and $\left\{\gamma_{n}\right\}$. If there exist $\varepsilon>0$ and an index $j$ such that $\left|\xi_{j}-\xi_{n}\right|>\varepsilon$ for all $n \neq j$, then $S$ is said to be associated with a measure having a separate mass point. In the case where the product has only one term, $S$ is called an atomic singular inner function.

In Section 3 we consider purely atomic singular inner functions. A useful auxiliary result for our purposes is a combination of the first corollary of [2, Theorem 5] and [27, Theorems 4.4.5 and 4.4.8]: If $0<p<\infty$ and $S$ is a singular inner function, then

$$
\frac{\int_{0}^{2 \pi}\left(1-\left|S\left(r e^{i t}\right)\right|\right)^{p} d t}{(1-r)^{p}} \gtrsim\left\{\begin{aligned}
1, & p<\frac{1}{2} \\
\log \left(\frac{e}{1-r}\right), & p=\frac{1}{2} \\
(1-r)^{1 / 2-p}, & p>\frac{1}{2}
\end{aligned}\right.
$$

for $0 \leq r<1$. An immediate consequence of this estimate and (1.1) is that there does not exist singular inner functions $S$ such that $S^{\prime} \in A_{\omega}^{p}$ if $\omega \in \widehat{\mathcal{D}}_{p}$ and either $p=\frac{1}{2}$ and $\int_{0}^{1} \omega(r) \log \left(\frac{1}{1-r}\right) d r=\infty$ or $p>\frac{1}{2}$ and $\int_{0}^{1} \omega(r)(1-r)^{\frac{1}{2}-p} d r=\infty$. Regarding (1.6), 
we will show that purely atomic singular inner functions associated with measures whose masses $\gamma_{n}$ satisfy $\sum_{n} \gamma_{n}^{\widehat{p}}<\infty$, where $\widehat{p}=\min \left\{\frac{1}{2}, p\right\}$, obey $\asymp$ instead of $\gtrsim$ only. Further, it will turn out that for $p \geq \frac{1}{2}$ these are the only singular inner functions satisfying $\asymp$ instead of $\gtrsim$ in (1.6). As a consequence of these deductions and (1.1), we obtain the following theorem which is the last of the main results of this study.

Theorem 3. Let $0<p<\infty$ and $\widehat{p}=\min \left\{\frac{1}{2}, p\right\}$. Let $\omega$ be a radial weight, and let $S$ be a purely atomic singular inner function satisfying $\sum_{n=1}^{\infty} \gamma_{n}^{\widehat{p}}<\infty$. Moreover, assume that either $\omega \in \widehat{\mathcal{D}}_{p}$ or $S$ is associated with a measure having a separate mass point.

(a) If $p<\frac{1}{2}$, then $S^{\prime} \in A_{\omega}^{p}$ and $\int_{\mathbf{D}}\left(\frac{1-|S(z)|^{2}}{1-|z|^{2}}\right)^{p} \omega(z) d A(z)<\infty$.

(b) If $p=\frac{1}{2}$, then the following statements are equivalent:

(i) $S^{\prime} \in A_{\omega}^{p}$;

(ii) $\int_{\mathbf{D}}\left(\frac{1-|S(z)|^{2}}{1-|z|^{2}}\right)^{p} \omega(z) d A(z)<\infty$;

(iii) $\int_{0}^{1} \omega(r) \log \left(\frac{1}{1-r}\right) d r<\infty$.

(c) If $p>\frac{1}{2}$, then the following statements are equivalent:

(i) $S^{\prime} \in A_{\omega}^{p}$;

(ii) $\int_{\mathbf{D}}\left(\frac{1-|S(z)|^{2}}{1-|z|^{2}}\right)^{p} \omega(z) d A(z)<\infty$;

(iii) $\int_{0}^{1} \omega(r)(1-r)^{\frac{1}{2}-p} d r<\infty$.

Theorem 3 is based on Theorems 8 and 10, to be proven in Section 3, which show that $M_{p}^{p}\left(r, S^{\prime}\right)$ and $\int_{0}^{2 \pi}\left(1-\left|S\left(r e^{i t}\right)\right|\right)^{p} d t /(1-r)^{p}$ are comparable under appropriate hypotheses. Since these results concern the $L^{p}$-means of $S^{\prime}$, they immediately give information on the question of when $S^{\prime}$ belongs to the Hardy space $H^{p}$. At this point it is also worth observing that for all inner functions $\Theta$ the quantities $\left\|\Theta^{\prime}\right\|_{H^{p}}^{p}$ and $\sup _{0<r<1} \int_{0}^{2 \pi}\left(1-\left|\Theta\left(r e^{i t}\right)\right|\right)^{p} d t /(1-r)^{p}$ are comparable, see, for example, [31, Theorem 2].

We have not found the statement of Theorem 3 even in the special case of the classical weighted Bergman spaces $A_{\alpha}^{p}$ in the existing literature. Although, by using the estimates for $\int_{0}^{2 \pi}\left(1-\left|S\left(r e^{i t}\right)\right|\right) d t$ and $M_{1 / 2}\left(r, S^{\prime}\right)$ established in [1, 5], where $S$ is as in Theorem 3, one may easily prove some particular cases of our theorem. Moreover, in view of the main result in [26] our result does not come as a surprise in the case of atomic singular inner functions.

\section{Blaschke products}

We begin with upper bounds for $\left\|B^{\prime}\right\|_{A_{\omega}^{p}}$ when $B$ is any Blaschke product. For short, we write $\omega \in \widehat{\mathcal{D}}_{\log }$ if

$$
\sup _{0<r<1}\left(\log \left(\frac{e}{1-r}\right) \widehat{\omega}(r)\right)^{-1} \int_{0}^{r} \log \left(\frac{e}{1-s}\right) \omega(s) d s<\infty .
$$

Proposition 4. Let $B$ be the Blaschke product associated with a sequence $\left\{z_{n}\right\}_{n=1}^{\infty}$, and let $\omega$ be a radial weight.

(a) If $0<p<\frac{1}{2}$, then $\left\|B^{\prime}\right\|_{A_{\omega}^{p}}^{p} \lesssim \sum_{n=1}^{\infty}\left(1-\left|z_{n}\right|\right)^{p}$. 
(b) If $p=\frac{1}{2}$ and $\omega \in \widehat{\mathcal{D}}_{\log }$, then

$$
\left\|B^{\prime}\right\|_{A_{\omega}^{p}}^{p} \lesssim \sum_{n=1}^{\infty} \frac{\widehat{\omega}\left(z_{n}\right)}{\left(1-\left|z_{n}\right|\right)^{p-1}} \log \frac{e}{1-\left|z_{n}\right|} .
$$

(c) If $\frac{1}{2}<p \leq 1$ and $\omega \in \widehat{\mathcal{D}}_{2 p-1}$, then

$$
\left\|B^{\prime}\right\|_{A_{\omega}^{p}}^{p} \lesssim \sum_{n=1}^{\infty} \frac{\widehat{\omega}\left(z_{n}\right)}{\left(1-\left|z_{n}\right|\right)^{p-1}} .
$$

(d) If $1<p<\infty$ and $\omega \in \widehat{\mathcal{D}}_{p} \cap \mathcal{J}_{p-1}$, then

$$
\left\|B^{\prime}\right\|_{A_{\omega}^{p}}^{p} \lesssim \sum_{n=1}^{\infty} \frac{\widehat{\omega}\left(z_{n}\right)}{\left(1-\left|z_{n}\right|\right)^{p-1}} .
$$

Proof. Since

$$
\frac{B^{\prime}(z)}{B(z)}=\sum_{n=1}^{\infty} \frac{\left|z_{n}\right|^{2}-1}{\left(1-\bar{z}_{n} z\right)\left(z_{n}-z\right)}
$$

we have

$$
\begin{aligned}
\left|B^{\prime}(z)\right| & =\left|\sum_{n=1}^{\infty} \frac{1-\left|z_{n}\right|^{2}}{\left(1-\bar{z}_{n} z\right)\left(z_{n}-z\right)}\right|\left|\prod_{k=1}^{\infty} \frac{\left|z_{k}\right|}{z_{k}} \frac{z_{k}-z}{1-\bar{z}_{k} z}\right| \\
& \leq \sum_{n=1}^{\infty} \frac{1-\left|z_{n}\right|^{2}}{\left|z_{n}-z\right|\left|1-\bar{z}_{n} z\right|} \frac{\left|z_{n}-z\right|}{\left|1-\bar{z}_{n} z\right|}\left|B_{n}(z)\right| \leq \sum_{n=1}^{\infty}\left|\varphi_{z_{n}}^{\prime}(z)\right|,
\end{aligned}
$$

where $B_{n}(z)=\prod_{k \neq n} \frac{\left|z_{k}\right|}{z_{k}} \frac{z_{k}-z}{1-\bar{z}_{k} z}$ and $\varphi_{a}(z)=\frac{a-z}{1-\bar{a} z}$ for all $a, z \in \mathbf{D}$. If $0<p \leq 1$, then $h(x)=x^{p}$ is sub-additive, and hence

$$
\int_{\mathbf{D}}\left|B^{\prime}(z)\right|^{p} \omega(z) d A(z) \leq \sum_{n=1}^{\infty}\left(1-\left|z_{n}\right|^{2}\right)^{p} \int_{\mathbf{D}} \frac{\omega(z)}{\left|1-\bar{z}_{n} z\right|^{2 p}} d A(z),
$$

where, by direct calculations,

$$
\int_{\mathbf{D}} \frac{\omega(z)}{\left|1-\bar{z}_{n} z\right|^{2 p}} d A(z) \asymp\left\{\begin{aligned}
1, & 0<p<\frac{1}{2}, \\
\int_{0}^{1} \log \frac{e}{1-\left|z_{n}\right| r} \omega(r) d r, & p=\frac{1}{2}, \\
\int_{0}^{1} \frac{\omega(r)}{\left(1-\left|z_{n}\right| r\right)^{2 p-1}} d r, & \frac{1}{2}<p \leq 1 .
\end{aligned}\right.
$$

The assertions in the cases (a)-(c) now follow by dividing the integrals into two parts, from zero to $\left|z_{n}\right|$ and the rest, then by estimating in a natural manner and finally using the hypotheses. If $p \geq 1$, then the Schwarz-Pick lemma and a similar deduction as in the case $p=1$ yield

$$
\begin{aligned}
\int_{\mathbf{D}}\left|B^{\prime}(z)\right|^{p} \omega(z) d A(z) & \leq \int_{\mathbf{D}}\left|B^{\prime}(z)\right| \frac{\omega(z)}{(1-|z|)^{p-1}} d A(z) \\
& \lesssim \sum_{n=1}^{\infty}\left(1-\left|z_{n}\right|\right) \int_{0}^{1} \frac{\omega(r)}{(1-r)^{p-1}\left(1-\left|z_{n}\right| r\right)} d r
\end{aligned}
$$

and the assertion (d) follows similarly as in the previous cases.

Let $N(f)(z)=\sup _{\zeta \in \Gamma(z)}|f(\zeta)|$ denote the maximal function related to the lens type regions

$$
\Gamma(z)=\left\{\zeta \in \mathbf{D}:|\arg z-\arg \zeta|<\frac{1}{2}\left(1-\left|\frac{\zeta}{z}\right|\right)\right\}, \quad z \in \mathbf{D} \backslash\{0\},
$$


with vertexes inside the disc. The Hardy-Littlewood maximal theorem [12, Theorem 3.1, p. 55] shows that $N: A_{\omega}^{p} \rightarrow L_{\omega}^{p}$ is bounded and there exists a constant $C>0$, independent of $p$, such that

$$
\|f\|_{A_{\omega}^{p}}^{p} \leq\|N(f)\|_{L_{\omega}^{p}}^{p} \leq C\|f\|_{A_{\omega}^{p}}^{p}, \quad f \in \mathcal{H}(\mathbf{D})
$$

see [28, Lemma 4.4] for details. With these preparations we are ready to prove our main result on Blaschke products.

Proof of Theorem 1. By Proposition 4 it suffices to show that $\sum_{n=1}^{\infty} \frac{\widehat{\omega}\left(z_{n}\right)}{\left(1-\left|z_{n}\right|\right)^{p-1}}$ is dominated by a constant times $\left\|B^{\prime}\right\|_{A_{\omega}^{p}}^{p}$. Note that for Proposition 4 we have to assume either $\frac{1}{2}<p \leq 1$ and $\omega \in \widehat{\mathcal{D}}_{2 p-1}$ or $1<p<\infty$ and $\omega \in \widehat{\mathcal{D}}_{p} \cap \mathcal{J}_{p-1}$. In the remaining part of the proof only the hypothesis $\omega \in \widehat{\mathcal{D}}_{p} \cap \mathcal{D}$ is needed. It is worth noting that this part uses some ideas from the proof of [31, Theorem 1].

Let $\left\{z_{n}\right\}_{n=1}^{\infty}=\bigcup_{j=1}^{M}\left\{z_{n}^{j}\right\}_{n=1}^{\infty}$, where each $\left\{z_{n}^{j}\right\}_{n=1}^{\infty}$ is separated with a separation constant $\delta_{j}$. Let $r<\min \left\{\delta_{j}: j=1, \ldots, M\right\}$ such that for given $j$, the $\operatorname{discs} \Delta\left(z_{n}^{j}\right)=$ $\left\{z \in \mathbf{D}:\left|z_{n}^{j}-z\right|<r\left(1-\left|z_{n}^{j}\right|\right)\right\}$ are pairwise disjoint. Then

$$
|B(z)| \leq \frac{\left|z-z_{n}^{j}\right|}{\left|1-\overline{z_{n}^{j}} z\right|} \leq \frac{\left|z-z_{n}^{j}\right|}{1-\left|z_{n}^{j}\right|}<r, \quad z \in \Delta\left(z_{n}^{j}\right),
$$

and hence

$$
\sup _{z \in \cup \Delta\left(z_{n}^{j}\right)}|B(z)| \leq r<1, \quad j=1, \ldots, M .
$$

Since $\omega \in \mathcal{D}$ by the hypothesis, $\widehat{\omega}$ is essentially constant in each disc $\Delta\left(z_{n}^{j}\right)$ by (1.2). This and the obvious inequality $1-|B(r \xi)| \leq \int_{r}^{1}\left|B^{\prime}(s \xi)\right| d s$, valid for almost every $\xi \in \mathbf{T}$, now yield

$$
\begin{aligned}
\sum_{n=1}^{\infty} \frac{\widehat{\omega}\left(z_{n}\right)}{\left(1-\left|z_{n}\right|\right)^{p-1}} & =\sum_{j=1}^{M} \sum_{n=1}^{\infty} \frac{\widehat{\omega}\left(z_{n}^{j}\right)}{\left(1-\left|z_{n}^{j}\right|\right)^{p-1}} \\
& \lesssim \sum_{j=1}^{M} \sum_{n=1}^{\infty} \int_{\Delta\left(z_{n}^{j}\right)}(1-|B(z)|)^{p} d A(z) \frac{\widehat{\omega}\left(z_{n}^{j}\right)}{\left(1-\left|z_{n}^{j}\right|\right)^{p+1}} \\
& \asymp \sum_{j=1}^{M} \sum_{n=1}^{\infty} \int_{\Delta\left(z_{n}^{j}\right)}(1-|B(z)|)^{p} \frac{\widehat{\omega}(z)}{(1-|z|)^{p+1}} d A(z) \\
& \leq M \int_{\mathbf{D}}(1-|B(z)|)^{p} \frac{\widehat{\omega}(z)}{(1-|z|)^{p+1}} d A(z) \\
& \leq M \int_{\mathbf{D}}\left(\int_{|z|}^{1}\left|B^{\prime}\left(s \frac{z}{|z|}\right)\right| d s\right)^{p} \frac{\widehat{\omega}(z)}{(1-|z|)^{p+1}} d A(z)
\end{aligned}
$$

Consider first the case $0<p \leq 1$. By [31, Lemma 4], the inner integral is dominated by a constant times

$$
\left(\int_{|z|}^{1} N\left(B^{\prime}\right)\left(s \frac{z}{|z|}\right)^{p}(1-s)^{p-1} d s\right)^{\frac{1}{p}} .
$$


This estimate together with Fubini's theorem and (2.1) gives

$$
\begin{aligned}
\sum_{n=1}^{\infty} \frac{\widehat{\omega}\left(z_{n}\right)}{\left(1-\left|z_{n}\right|\right)^{p-1}} & \lesssim \int_{\mathbf{D}}\left|B^{\prime}(z)\right|^{p}(1-|z|)^{p-1}\left(\int_{0}^{|z|} \frac{\widehat{\omega}(s)}{(1-s)^{p+1}} d s\right) d A(z) \\
& =\int_{\mathbf{D}}\left|B^{\prime}(z)\right|^{p} d \mu_{p, \omega}(z)
\end{aligned}
$$

where

$$
d \mu_{p, \omega}(z)=(1-|z|)^{p-1}\left(\int_{0}^{|z|} \frac{\widehat{\omega}(s)}{(1-s)^{p+1}} d s\right) d A(z) .
$$

The right-hand side is bounded by a constant times $\left\|B^{\prime}\right\|_{A_{\omega}^{p}}^{p}$ if $A_{\omega}^{p}$ is continuously embedded into $L_{\mu_{p, \omega}}^{p}$, that is, if $\mu_{p, \omega}$ is a $p$-Carleson measure for $A_{\omega}^{p}$. By [29, Theorem 1] this is the case if (and only if) $\mu_{p, \omega}(S(a)) \lesssim \omega(S(a))$ for all Carleson squares $S(a)=\left\{z \in \mathbf{D}:|\arg z-\arg a|<\frac{1-|a|}{2},|z| \geq|a|\right\}$ with $a \in \mathbf{D} \backslash\{0\}$. Since both $\mu_{p, \omega}$ and $\omega$ are radial, this condition is equivalent to

$$
\int_{r}^{1}(1-t)^{p-1}\left(\int_{0}^{t} \frac{\widehat{\omega}(s)}{(1-s)^{p+1}} d s\right) d t \lesssim \widehat{\omega}(r), \quad 0<r<1 .
$$

Fubini's theorem shows that the left-hand side equals to

$$
\frac{1}{p}\left((1-r)^{p} \int_{0}^{r} \frac{\widehat{\omega}(s)}{(1-s)^{p+1}} d s+\int_{r}^{1} \frac{\widehat{\omega}(s)}{1-s} d s\right),
$$

where, by an integration by parts and the hypothesis $\omega \in \widehat{\mathcal{D}}_{p}$,

$$
\begin{aligned}
\int_{0}^{r} \frac{\widehat{\omega}(s)}{(1-s)^{p+1}} d s & =\frac{1}{p}\left(\frac{\widehat{\omega}(r)}{(1-r)^{p}}-\widehat{\omega}(0)+\int_{0}^{r} \frac{\omega(s)}{(1-s)^{p}} d s\right) \\
& \leq \frac{1}{p}\left(\frac{\widehat{\omega}(r)}{(1-r)^{p}}-\widehat{\omega}(0)+\widehat{\mathcal{D}}_{p}(\omega) \frac{\widehat{\omega}(r)}{(1-r)^{p}}\right)
\end{aligned}
$$

and

$$
\int_{r}^{1} \frac{\widehat{\omega}(s)}{1-s} d s=\int_{r}^{1} \frac{\widehat{\omega}(s)}{(1-s)^{\beta}} \frac{d s}{(1-s)^{1-\beta}} \lesssim \frac{\widehat{\omega}(r)}{(1-r)^{\beta}} \int_{r}^{1} \frac{d s}{(1-s)^{1-\beta}} \asymp \widehat{\omega}(r)
$$

by the first inequality in (1.2). It follows that (2.2) is satisfied, and hence the case $0<p \leq 1$ is proved.

Let now $1<p<\infty$ and $\omega \in \widehat{\mathcal{D}}_{p} \cap \mathcal{D}$. Two integrations by parts show that the condition $\omega \in \widehat{\mathcal{D}}_{p}$ is self-improving in the sense that if $\omega \in \widehat{\mathcal{D}}_{p}$, then $\omega \in \widehat{\mathcal{D}}_{p-\varepsilon}$ for all $\varepsilon>0$ sufficiently small, see the proof of [31, Lemma 3] for details. Hence we may choose $\varepsilon=\varepsilon(p, \omega) \in(0, p-1)$ such that $\omega \in \widehat{\mathcal{D}}_{p-\varepsilon}$, and define $h(z)=(1-|z|)^{\frac{p-1-\varepsilon}{p}}$. Then Hölder's inequality and Fubini's theorem yield

$$
\begin{aligned}
\int_{\mathbf{D}} & \left(\int_{|z|}^{1}\left|B^{\prime}\left(s \frac{z}{|z|}\right)\right| d s\right)^{p} \frac{\widehat{\omega}(z)}{(1-|z|)^{p+1}} d A(z) \\
& \leq \int_{\mathbf{D}} \int_{|z|}^{1}\left|B^{\prime}\left(s \frac{z}{|z|}\right)\right|^{p} h(s)^{p} d s\left(\int_{|z|}^{1} \frac{d t}{h(t)^{p^{\prime}}}\right)^{p-1} \frac{\widehat{\omega}(z)}{(1-|z|)^{p+1}} d A(z) \\
& =\int_{0}^{1} \int_{0}^{2 \pi}\left|B^{\prime}\left(s e^{i \theta}\right)\right|^{p} d \theta h(s)^{p} \int_{0}^{s}\left(\int_{r}^{1} \frac{d t}{h(t)^{p^{\prime}}}\right)^{p-1} \frac{\widehat{\omega}(r)}{(1-r)^{p+1}} r d r d s
\end{aligned}
$$




$$
\begin{aligned}
& \asymp \int_{\mathbf{D}}\left|B^{\prime}(z)\right|^{p} h(z)^{p} \int_{0}^{|z|}\left(\int_{r}^{1} \frac{d t}{h(t)^{p^{\prime}}}\right)^{p-1} \frac{\widehat{\omega}(r)}{(1-r)^{p+1}} d r d A(z) \\
& \asymp \int_{\mathbf{D}}\left|B^{\prime}(z)\right|^{p}(1-|z|)^{p-1-\varepsilon}\left(\int_{0}^{|z|} \frac{\widehat{\omega}(r)}{(1-r)^{p+1-\varepsilon}} d r\right) d A(z) .
\end{aligned}
$$

Since the $p$-Carleson measures for $A_{\omega}^{p}$ are independent of $p$ by [29, Theorem 1], (2.2) with $p$ replaced by $p-\varepsilon$ implies that $\mu_{p-\varepsilon, \omega}$ is a $p$-Carleson measure for $A_{\omega}^{p}$. The assertion in the case $1<p<\infty$ follows, and the proof is complete.

The Blaschke product $B$ with the zero-sequence $\left\{z_{n}\right\}$ is called a Carleson-Newman Blaschke product if the measure

$$
\mu=\sum_{n=1}^{\infty}\left(1-\left|z_{n}\right|\right) \delta_{z_{n}}
$$

is a $p$-Carleson measure for $H^{p}$. This is equivalent to $\left\{z_{n}\right\}$ being a finite union of uniformly separated sequences which is the same as $B$ being a finite product of interpolating Blaschke products. An equivalent quantitative condition is

$$
\sup _{a \in \mathbf{D}} \sum_{n=1}^{\infty}\left(1-\left|\varphi_{a}\left(z_{n}\right)\right|\right)<\infty
$$

see $[12,23,24]$. Recall that $\left\{z_{n}\right\}_{n=1}^{\infty}$ is uniformly separated, if there exists a constant $\delta>0$ such that

$$
\inf _{n \in \mathbf{N}} \prod_{k \neq n}\left|\frac{z_{k}-z_{n}}{1-\bar{z}_{k} z_{n}}\right|=\delta .
$$

The following result shows that in Theorem 1 we may omit the hypothesis $\omega \in \mathcal{D}$ if $1 \leq p<\infty$ and $B$ is a Carleson-Newman Blaschke product.

Proposition 5. Let $1 \leq p<\infty$ and $\omega \in \widehat{\mathcal{D}}_{p}$, and let $B$ be the Carleson-Newman Blaschke product associated with $\left\{z_{n}\right\}_{n=1}^{\infty}$. Then

$$
\sum_{n=1}^{\infty} \frac{\widehat{\omega}\left(z_{n}\right)}{\left(1-\left|z_{n}\right|\right)^{p-1}} \lesssim\left\|B^{\prime}\right\|_{A_{\omega}^{p}}^{p} .
$$

Proof. It is well known that the Carleson-Newman Blaschke product $B$ satisfies

$$
1-|B(z)|^{2} \gtrsim \sum_{n=1}^{\infty}\left(1-\left|\varphi_{z_{n}}(z)\right|^{2}\right), \quad z \in \mathbf{D} .
$$

We sketch a proof of this fact for the convenience of the reader. Since $1-r^{2} \leq$ $-2 \log r$ for $0<r \leq 1,2 \log |B(z)| \leq-\sum_{n=1}^{\infty}\left(1-\left|\varphi_{z_{n}}(z)\right|^{2}\right)$, and hence $|B(z)|^{2} \leq$ $\exp \left(-\sum_{n=1}^{\infty}\left(1-\left|\varphi_{z_{n}}(z)\right|^{2}\right)\right)$. This together with (2.3) and the fact that $\left(1-e^{-x}\right) / x$ is decreasing yields (2.4). By combining (1.1) and (2.4) we deduce

$$
\begin{aligned}
\left\|B^{\prime}\right\|_{A_{\omega}^{p}}^{p} & \gtrsim \int_{\mathbf{D}} \sum_{n=1}^{\infty}\left(1-\left|\varphi_{z_{n}}(z)\right|^{2}\right)^{p} \frac{\omega(z)}{(1-|z|)^{p}} d A(z) \\
& \asymp \sum_{n=1}^{\infty}\left(1-\left|z_{n}\right|\right)^{p} \int_{0}^{1} \frac{\omega(s)}{\left(1-\left|z_{n}\right| s\right)^{2 p-1}} d s \gtrsim \sum_{n=1}^{\infty} \frac{\widehat{\omega}\left(z_{n}\right)}{\left(1-\left|z_{n}\right|\right)^{p-1}},
\end{aligned}
$$

and the assertion is proved. 
If $\Theta$ is an inner function, then there exists a Blaschke product $B_{\Theta}$ associated with a uniformly separated sequence $\left\{z_{n}\right\}$ such that $1-|\Theta(z)| \asymp 1-\left|B_{\Theta}(z)\right|$ for all $z \in \mathbf{D}[7,8] . \quad B_{\Theta}$ is called an approximating Blaschke product of $\Theta$. By using Proposition 5, we obtain the following result.

Corollary 6. Let $1<p<\infty$ and $\omega \in \widehat{\mathcal{D}}_{p}$ such that $\widehat{\omega}(r)(1-r)^{1-p} \gtrsim 1$, as $r \rightarrow 1^{-}$, and let $\Theta$ be an inner function. Then $\Theta^{\prime} \in A_{\omega}^{p}$ if and only if $\Theta$ is a finite Blaschke product.

Proof. Since $\Theta^{\prime} \in H^{\infty}$ if $\Theta$ is a finite Blaschke product, it suffices to prove the "only if" part of the assertion. Let $\Theta$ be an inner function and assume first that its approximating Blaschke product $B_{\Theta}$ has infinitely many zeros $\left\{z_{n}\right\}_{n=1}^{\infty}$. Then (1.1), Proposition 5 and the hypothesis $\widehat{\omega}(r)(1-r)^{1-p} \gtrsim 1$, as $r \rightarrow 1^{-}$, yield

$$
\begin{aligned}
\left\|\Theta^{\prime}\right\|_{A_{\omega}^{p}}^{p} & \asymp \int_{\mathbf{D}}\left(\frac{1-|\Theta(z)|^{2}}{1-|z|^{2}}\right)^{p} \omega(z) d A(z) \asymp \int_{\mathbf{D}}\left(\frac{1-\left|B_{\Theta}(z)\right|^{2}}{1-|z|^{2}}\right)^{p} \omega(z) d A(z) \\
& \asymp\left\|B_{\Theta}^{\prime}\right\|_{A_{\omega}^{p}}^{p} \gtrsim \sum_{n=1}^{\infty} \frac{\widehat{\omega}\left(z_{n}\right)}{\left(1-\left|z_{n}\right|\right)^{p-1}}=\infty .
\end{aligned}
$$

Hence $\Theta^{\prime} \in A_{\omega}^{p}$ only if $B_{\Theta}$ is a finite Blaschke product. But if $B_{\Theta}$ has finitely many zeros, then

$$
\left|\Theta^{\prime}(z)\right| \leq \frac{1-|\Theta(z)|^{2}}{1-|z|^{2}} \asymp \frac{1-\left|B_{\Theta}(z)\right|^{2}}{1-|z|^{2}} \asymp\left|B_{\Theta}^{\prime}(z)\right|, \quad|z| \rightarrow 1^{-}
$$

and hence $\Theta$ is continuous up to the boundary [10, Theorem 3.11]. Therefore $\Theta$ is a finite Blaschke product, and the assertion is proved.

We next establish a generalization of [6, Corollary 2] and [19, Theorem 7(b)]. For $q>0$ and a weight $\omega$, we write $\omega_{q}(z)=\omega(z)(1-|z|)^{q}$ for all $z \in \mathbf{D}$. Corollary 7 shows that, under appropriate hypotheses, the quantities $\left\|\Theta^{\prime}\right\|_{A_{\omega}^{p}}$ and $\left\|\Theta^{\prime}\right\|_{A_{\omega q}^{p+q}}$ are finite at the same time for each inner function $\Theta$.

Corollary 7. Let $\frac{1}{2}<p<\infty, 0<q<\infty$ and $\omega \in \mathcal{D}$, and let $\Theta$ be an inner function. If

(a) $1<p<\infty$ and $\omega \in \widehat{\mathcal{D}}_{p} \cap \mathcal{J}_{p-1}$, or

(b) $p+q \leq 1$ and $\omega \in \widehat{\mathcal{D}}_{2 p-1}$, or

(c) $1<p+q \leq 1+q$ and $\omega \in \widehat{\mathcal{D}}_{2 p-1} \cap \mathcal{J}_{p-1}$,

then $\left\|\Theta^{\prime}\right\|_{A_{\omega}^{p}}^{p} \asymp\left\|\Theta^{\prime}\right\|_{A_{\omega_{q}}^{p+q}}^{p+q}$.

Proof. We begin with showing that if $\omega \in \mathcal{D}$ and $0<q<\infty$, then

$$
\widehat{\omega_{q}}(z) \asymp \widehat{\omega}(z)(1-|z|)^{q}, \quad z \in \mathbf{D} .
$$

Since for each radial $\omega$ we have $\widehat{\omega}_{q}(z) \leq \widehat{\omega}(z)(1-|z|)^{q}$ for all $z \in \mathbf{D}$, it suffices to show that $\widehat{\omega_{q}}(r) \gtrsim \widehat{\omega}(r)(1-r)^{q}$ for all $0 \leq r<1$. To see this, let $C=C(\omega) \geq 1$, $\alpha=\alpha(\omega)>0$ and $\beta=\beta(\omega) \geq \alpha$ be the constants appearing in (1.2). Let $0 \leq r<1$ and choose $a=a(\omega)$ such that $1-C^{-1 / \alpha}<a<1$. Set $r_{0}=r$ and $r_{n+1}=r_{n}+a\left(1-r_{n}\right)$ 
for all $n \in \mathbf{N} \cup\{0\}$. Then $r_{n} \rightarrow 1^{-}$, as $n \rightarrow \infty$, and hence (1.2) yields

$$
\begin{aligned}
\widehat{\omega}_{q}(r) & =\sum_{n=0}^{\infty} \int_{r_{n}}^{r_{n+1}} \omega(s)(1-s)^{q} d s \geq \sum_{n=0}^{\infty}\left(1-r_{n+1}\right)^{q}\left(\widehat{\omega}\left(r_{n}\right)-\widehat{\omega}\left(r_{n+1}\right)\right) \\
& =(1-r)^{q} \sum_{n=0}^{\infty}(1-a)^{q(n+1)}\left(\widehat{\omega}\left(r_{n}\right)-\widehat{\omega}\left(r_{n+1}\right)\right) \\
& \geq(1-r)^{q} \sum_{n=0}^{\infty}(1-a)^{q(n+1)} \widehat{\omega}\left(r_{n}\right)\left(1-C\left(\frac{1-r_{n+1}}{1-r_{n}}\right)^{\alpha}\right) \\
& =\left(1-C(1-a)^{\alpha}\right)(1-r)^{q} \sum_{n=0}^{\infty}(1-a)^{q(n+1)} \widehat{\omega}\left(r_{n}\right) \\
& \geq C^{-1}\left(1-C(1-a)^{\alpha}\right) \widehat{\omega}(r)(1-r)^{q} \sum_{n=0}^{\infty}(1-a)^{q(n+1)}\left(\frac{1-r_{n}}{1-r}\right)^{\beta} \\
& =C^{-1}\left(1-C(1-a)^{\alpha}\right) \widehat{\omega}(r)(1-r)^{q} \sum_{n=0}^{\infty}(1-a)^{n(q+\beta)+q} \asymp \widehat{\omega}(r)(1-r)^{q},
\end{aligned}
$$

and (2.5) follows.

Let $B_{\Theta}$ be the approximating Blaschke product of $\Theta$ with zeros $\left\{z_{n}\right\}_{n=1}^{\infty}$. Let first $1<p<\infty$. By using (2.5) it is easy to see that the conditions $\omega \in \widehat{\mathcal{D}}_{p}$ and $\omega \in \mathcal{J}_{p-1}$ are equivalent to $\omega_{q} \in \widehat{\mathcal{D}}_{p+q}$ and $\omega_{q} \in \mathcal{J}_{p+q-1}$, respectively. Therefore (1.1), Theorem 1 and (2.5) yield

$$
\left\|\Theta^{\prime}\right\|_{A_{\omega}^{p}}^{p} \asymp\left\|B_{\Theta}^{\prime}\right\|_{A_{\omega}^{p}}^{p} \asymp \sum_{n=1}^{\infty} \frac{\widehat{\omega}\left(z_{n}\right)}{\left(1-\left|z_{n}\right|\right)^{p-1}} \asymp \sum_{n=1}^{\infty} \frac{\widehat{\omega}_{q}\left(z_{n}\right)}{\left(1-\left|z_{n}\right|\right)^{p+q-1}} \asymp\left\|B_{\Theta}^{\prime}\right\|_{A_{\omega q}^{p+q}}^{p+q} \asymp\left\|\Theta^{\prime}\right\|_{A_{\omega_{q}}^{p+q}}^{p+q} \text {, }
$$

and thus the assertion is proved for $1<p<\infty$. The other two cases follow in a similar manner.

We end this section with the proof of Theorem 2 .

Proof of Theorem 2. Let $\frac{1}{2}<p<\infty$ and $\varepsilon>0$ be as in the statement of the theorem. Assume, without loss of generality, that $\left\{z_{n}\right\}$ is ordered by increasing moduli and $z_{n} \neq 0$ for all $n$. An integration by parts shows that $\omega \in \widehat{\mathcal{D}}_{p}$ if and only if

$$
\frac{(1-r)^{p}}{\widehat{\omega}(r)} \int_{0}^{r} \frac{\widehat{\omega}(s)}{(1-s)^{p+1}} d s \asymp 1, \quad r \rightarrow 1^{-} .
$$

But since (1.4) is satisfied by the hypothesis,

$$
\int_{0}^{r} \frac{\widehat{\omega}(s)}{(1-s)^{p+1}} d s \lesssim \frac{\widehat{\omega}(r)}{(1-r)^{p-\varepsilon}} \int_{0}^{r} \frac{d s}{(1-s)^{p+1-p+\varepsilon}} \asymp \frac{\widehat{\omega}(r)}{(1-r)^{p}}, \quad 0 \leq r<1,
$$

and thus $\omega \in \widehat{\mathcal{D}}_{p}$. Further, by the proof of $[3$, Theorem 6$]$,

$$
\frac{1-|B(z)|^{2}}{1-|z|^{2}}=\sum_{n=1}^{\infty}\left|B_{n}(z)\right|^{2} \frac{1-\left|z_{n}\right|^{2}}{\left|1-\bar{z}_{n} z\right|^{2}}, \quad z \in \mathbf{D},
$$

where

$$
B_{n}(z)=\prod_{j=1}^{n-1} \frac{z_{j}-z}{1-\bar{z}_{j} z}, \quad n \in \mathbf{N}, \quad z \in \mathbf{D}
$$


and hence (1.1) implies

$$
\left\|B^{\prime}\right\|_{A_{\omega}^{p}}^{p} \asymp \int_{\mathbf{D}}\left(\sum_{n=1}^{\infty}\left|B_{n}(z)\right|^{2} \frac{1-\left|z_{n}\right|}{\left|1-\bar{z}_{n} z\right|^{2}}\right)^{p} \omega(r) d A(z) .
$$

We next estimate $\left|B_{n}\right|$ appropriately downwards close to the boundary. To do this, let

$$
\rho_{\varepsilon, \gamma}(z)=\widehat{\omega}(z)^{\frac{1}{\varepsilon+\min \{0,1-p\}}}(1-|z|)^{\gamma-1}, \quad z \in \mathbf{D},
$$

and set $\gamma_{0}=\inf \left\{\gamma \geq 0: \sum_{n=1}^{\infty} \rho_{n}\left(1-\left|z_{n}\right|\right)<\infty\right\}$, where $\rho_{n}=\rho_{\varepsilon, \gamma}\left(z_{n}\right)$. Since $\left\{z_{n}\right\}$ is a Blaschke sequence, $\gamma_{0} \leq 1$. Let $\gamma>\gamma_{0}=\gamma_{0}(\varepsilon)$ so that $\sum_{n=1}^{\infty} \rho_{n}\left(1-\left|z_{n}\right|\right)$ converges. As in the proof of [32, Theorem 1], note that

$$
\frac{r-\left|z_{j}\right|}{1-\left|z_{j}\right| r} \geq\left|z_{j}\right|^{\rho} \Longleftrightarrow r \geq \frac{\left|z_{j}\right|+\left|z_{j}\right|^{\rho}}{1+\left|z_{j}\right|^{\rho+1}}, \quad 0<r<1, \quad 0<\rho<\infty,
$$

and let

$$
r_{j}=\frac{\left|z_{j}\right|+\left|z_{j}\right|^{\rho_{j}}}{1+\left|z_{j}\right|^{\rho_{j}+1}}=1-\frac{\left(1-\left|z_{j}\right|\right)\left(1-\left|z_{j}\right|^{\rho_{j}}\right)}{1+\left|z_{j}\right|^{\rho_{j}+1}}, \quad j \in \mathbf{N} .
$$

Then $\left|z_{j}\right|<r_{j}<1$ for all $j \in \mathbf{N}$. Moreover, for $|z| \geq R_{n}=\max _{1 \leq j \leq n} r_{j}$, we have

$$
\begin{aligned}
\left|B_{n}(z)\right| & \geq \prod_{j=1}^{n-1} \frac{|z|-\left|z_{j}\right|}{1-\left|z_{j}\right||z|} \geq \prod_{j=1}^{n-1} \frac{R_{n}-\left|z_{j}\right|}{1-\left|z_{j}\right| R_{n}} \geq \prod_{j=1}^{n-1}\left|z_{j}\right|^{\rho_{j}} \geq \prod_{j=1}^{\infty}\left|z_{j}\right|^{\rho_{j}} \\
& =\exp \left(\sum_{j=1}^{\infty} \rho_{j} \log \left|z_{j}\right|\right) \geq \exp \left(-\sum_{j=1}^{\infty} \frac{\rho_{j}\left(1-\left|z_{j}\right|\right)}{\left|z_{j}\right|}\right) \gtrsim 1 .
\end{aligned}
$$

Let $\frac{1}{2}<p \leq 1$. Then (2.6), Minkowski's inequality and (2.7) yield

$$
\begin{aligned}
\left\|B^{\prime}\right\|_{A_{\omega}^{p}} & \gtrsim \sum_{n=1}^{\infty}\left(\int_{\mathbf{D}}\left|B_{n}(z)\right|^{2 p} \frac{\left(1-\left|z_{n}\right|\right)^{p}}{\left|1-\bar{z}_{n} z\right|^{2 p}} \omega(z) d A(z)\right)^{\frac{1}{p}} \\
& \gtrsim \sum_{n=1}^{\infty}\left(\int_{\mathbf{D} \backslash D\left(0, R_{n}\right)} \frac{\left(1-\left|z_{n}\right|\right)^{p}}{\left|1-\bar{z}_{n} z\right|^{2 p}} \omega(z) d A(z)\right)^{\frac{1}{p}} \\
& \asymp \sum_{n=1}^{\infty}\left(1-\left|z_{n}\right|\right)\left(\int_{R_{n}}^{1} \frac{\omega(r) d r}{\left(1-\left|z_{n}\right| r\right)^{2 p-1}}\right)^{\frac{1}{p}} .
\end{aligned}
$$

Recall that $\left|z_{j}\right|<r_{j} \leq R_{j}$, and hence

$$
\begin{aligned}
\left\|B^{\prime}\right\|_{A_{\omega}^{p}} & \gtrsim \sum_{n=1}^{\infty}\left(1-\left|z_{n}\right|\right)^{\frac{1}{p}-1} \widehat{\omega}\left(R_{n}\right)^{\frac{1}{p}} \gtrsim \sum_{n=1}^{\infty}\left(1-\left|z_{n}\right|\right)^{\frac{1}{p}-1} \widehat{\omega}\left(z_{n}\right)^{\frac{1}{p}}\left(\frac{1-R_{n}}{1-\left|z_{n}\right|}\right)^{\frac{p-\varepsilon}{p}} \\
& =\sum_{n=1}^{\infty}\left(1-\left|z_{n}\right|\right)^{\frac{1}{p}-1} \widehat{\omega}\left(z_{n}\right)^{\frac{1}{p}}\left(\frac{\inf _{1 \leq j \leq n}\left(1-r_{j}\right)}{1-\left|z_{n}\right|}\right)^{\frac{p-\varepsilon}{p}} .
\end{aligned}
$$

Since $1-r_{j} \geq\left(1-\left|z_{j}\right|\right)\left(1-\left|z_{j}\right|^{\rho_{j}}\right) / 2$ and $\left\{z_{n}\right\}_{n=1}^{\infty}$ is ordered by increasing moduli, we obtain

$$
\begin{aligned}
\left\|B^{\prime}\right\|_{A_{\omega}^{p}} & \gtrsim \sum_{n=1}^{\infty}\left(1-\left|z_{n}\right|\right)^{\frac{1}{p}-1} \widehat{\omega}\left(z_{n}\right)^{\frac{1}{p}}\left(\frac{\inf _{1 \leq j \leq n}\left(1-\left|z_{j}\right|\right)\left(1-\left|z_{j}\right|^{\rho_{j}}\right)}{1-\left|z_{n}\right|}\right)^{\frac{p-\varepsilon}{p}} \\
& \geq \sum_{n=1}^{\infty}\left(1-\left|z_{n}\right|\right)^{\frac{1}{p}-1} \widehat{\omega}\left(z_{n}\right)^{\frac{1}{p}}\left(\inf _{1 \leq j \leq n}\left(1-\left|z_{j}\right|^{\rho_{j}}\right)\right)^{\frac{p-\varepsilon}{p}}
\end{aligned}
$$


Now that $\sum_{j=1}^{\infty} \rho_{j}\left(1-\left|z_{j}\right|\right)$ converges and $z_{j} \neq 0$ for all $j$, there exists $\delta>0$ such that

$$
\inf _{j \in \mathbf{N}}\left|z_{j}\right|^{\rho_{j}}=\inf _{j \in \mathbf{N}}\left(\left|z_{j}\right|^{\left(1-\left|z_{j}\right|\right)^{-1}}\right)^{\rho_{j}\left(1-\left|z_{j}\right|\right)} \geq \delta
$$

Therefore

$$
\begin{aligned}
\left\|B^{\prime}\right\|_{A_{\omega}^{p}} & \gtrsim \sum_{n=1}^{\infty}\left(1-\left|z_{n}\right|\right)^{\frac{1}{p}-1} \widehat{\omega}\left(z_{n}\right)^{\frac{1}{p}}\left(\inf _{1 \leq j \leq n}\left|z_{j}\right|^{\rho_{j}} \log \frac{1}{\left|z_{j}\right|^{\rho_{j}}}\right)^{\frac{p-\varepsilon}{p}} \\
& \gtrsim \sum_{n=1}^{\infty}\left(1-\left|z_{n}\right|\right)^{\frac{1}{p}-1} \widehat{\omega}\left(z_{n}\right)^{\frac{1}{p}}\left(\inf _{1 \leq j \leq n} \rho_{j}\left(1-\left|z_{j}\right|\right)\right)^{\frac{p-\varepsilon}{p}} \\
& \geq \sum_{n=1}^{\infty}\left(1-\left|z_{n}\right|\right)^{\frac{1+\gamma(p-\varepsilon)-p}{p}} \widehat{\omega}\left(z_{n}\right)^{\frac{1}{\varepsilon}}
\end{aligned}
$$

If $B^{\prime} \in A_{\omega}^{p}$, then $1+\gamma(p-\varepsilon)-p>p \gamma_{0}$, and by letting $\gamma \rightarrow \gamma_{0}$, we deduce $\gamma_{0} \leq \frac{1-p}{\varepsilon}$. The assertion in the case $\frac{1}{2}<p \leq 1$ follows.

Let $p>1$. Then we can drop $p$ inside the sum in (2.6) without using Minkowski's inequality. Hence, by deducting as above, we obtain

$$
\left\|B^{\prime}\right\|_{A_{\omega}^{p}}^{p} \gtrsim \sum_{n=1}^{\infty}\left(1-\left|z_{n}\right|\right)^{1-p} \widehat{\omega}\left(R_{n}\right) \gtrsim \sum_{n=1}^{\infty}\left(1-\left|z_{n}\right|\right)^{1-p} \widehat{\omega}\left(z_{n}\right)\left(\inf _{1 \leq j \leq n} \rho_{j}\left(1-\left|z_{j}\right|\right)\right)^{p-\varepsilon} .
$$

Since the left-hand inequality of (1.5) is equivalent with the asymptotic inequality

$$
\widehat{\omega}(r)^{\frac{1}{1+\varepsilon-p}}(1-r)^{\gamma} \gtrsim \widehat{\omega}(t)^{\frac{1}{1+\varepsilon-p}}(1-t)^{\gamma}, \quad 0 \leq r \leq t<1
$$

we have

$$
\left\|B^{\prime}\right\|_{A_{\omega}^{p}}^{p} \gtrsim \sum_{n=1}^{\infty}\left(1-\left|z_{n}\right|\right)^{1+\gamma(p-\varepsilon)-p} \widehat{\omega}\left(z_{n}\right)^{\frac{1}{1+\varepsilon-p}} .
$$

If $B^{\prime} \in A_{\omega}^{p}$, then $1+\gamma(p-\varepsilon)-p>\gamma_{0}$, and by letting $\gamma \rightarrow \gamma_{0}$, we deduce $\gamma_{0} \leq \frac{1-p}{1+\varepsilon-p}$. The assertion in the case $1<p<\infty$ follows, and the proof is complete.

\section{Purely atomic singular inner functions}

Recall that purely atomic singular inner functions are of the form

$$
S(z)=\prod_{n} \exp \left(\gamma_{n} \frac{z+\xi_{n}}{z-\xi_{n}}\right)=\exp \left(\sum_{n} \gamma_{n} \frac{z+\xi_{n}}{z-\xi_{n}}\right), \quad z \in \mathbf{D},
$$

where $\xi_{n} \in \mathbf{T}$ are distinct points and $\sum_{n} \gamma_{n}<\infty$. If the product has only one term, with $\gamma_{1}=\gamma$ and $\xi_{1}=\xi$, then we write $S=S_{\gamma, \xi}$.

Theorem 8. Let $0<p<\infty$ and $\widehat{p}=\min \left\{\frac{1}{2}, p\right\}$. Let $S$ be the purely atomic singular inner function associated with $\left\{\xi_{n}\right\}$ and $\gamma=\left\{\gamma_{n}\right\} \in \ell^{\widehat{p}}$. Then

$$
\frac{\int_{0}^{2 \pi}\left(1-\left|S\left(r e^{i t}\right)\right|\right)^{p} d t}{(1-r)^{p}} \asymp h_{p}(r)=\left\{\begin{aligned}
1, & p<\frac{1}{2} \\
\log \left(\frac{1}{1-r}\right), & p=\frac{1}{2} \\
(1-r)^{1 / 2-p}, & p>\frac{1}{2}
\end{aligned}\right.
$$

for $\frac{1}{2}<r<1$. 
Proof. By (1.6), it suffices to show that $\int_{0}^{2 \pi}\left(1-\left|S\left(r e^{i t}\right)\right|\right)^{p} d t \lesssim(1-r)^{p} h_{p}(r)$. We begin with an estimate for $S=S_{\gamma, \xi}$, where $\xi=e^{i \theta}$. Since

$$
\begin{aligned}
\left|1-e^{i s}\right|^{2} & =2(1-\cos s)=2 s^{2} \sum_{k=1}^{\infty}(-1)^{k-1} \frac{s^{2(k-1)}}{(2 k) !} \\
& \geq 2 s^{2}\left(\frac{1}{2}-\frac{\pi^{2}}{4 !}+\frac{\pi^{4}}{6 !}-\frac{\pi^{6}}{8 !}\right) \geq \frac{s^{2}}{3}, \quad-\pi \leq s \leq \pi,
\end{aligned}
$$

we obtain

$$
\begin{aligned}
\int_{0}^{2 \pi}\left(1-\left|S_{\gamma, \xi}\left(r e^{i t}\right)\right|\right)^{p} d t & =\int_{0}^{2 \pi}\left(1-\exp \left(-\gamma \frac{1-r^{2}}{\left|1-r e^{i(t-\theta)}\right|^{2}}\right)\right)^{p} d t \\
& =2 \int_{0}^{\pi}\left(1-\exp \left(-\gamma \frac{1-r^{2}}{\left|1-r e^{i s}\right|^{2}}\right)\right)^{p} d s \\
& =2 \int_{0}^{\pi}\left(1-\exp \left(-\gamma \frac{1-r^{2}}{(1-r)^{2}+r\left|1-e^{i s}\right|^{2}}\right)\right)^{p} d s \\
& \leq 2 \int_{0}^{\pi}\left(1-\exp \left(-2 \gamma \frac{1-r}{(1-r)^{2}+\frac{r s^{2}}{3}}\right)\right)^{p} d s \\
& =2 \sqrt{\frac{3}{r}} \gamma^{\frac{1}{2}}(1-r)^{\frac{1}{2}} \int_{\frac{2 \gamma(1-r)}{(1-r)^{2}+r \pi^{2} / 3}}^{\frac{2 \gamma}{1-r}} \frac{\left(1-e^{\frac{3}{2}}\left(2-\frac{x(1-r)}{\gamma}\right)^{p} d x\right.}{\frac{1}{2}} \\
& \leq 2 \sqrt{6} \gamma^{\frac{1}{2}}(1-r)^{\frac{1}{2}} \int_{\frac{\gamma(1-r)}{2}}^{\frac{2 \gamma}{1-r}} \frac{\left(1-e^{-x}\right)^{p} d x}{x^{\frac{3}{2}}\left(2-\frac{x(1-r)}{\gamma}\right)^{\frac{1}{2}}}, \quad \frac{1}{2}<r<1 .
\end{aligned}
$$

To prove the general case, we may assume that $\left\{\gamma_{n}\right\}_{n=1}^{\infty}$ is non-increasing. Write $S_{n}=S_{\gamma_{n}, \xi_{n}}$ for short. If $\int_{0}^{2 \pi}\left(1-\left|S\left(r e^{i t}\right)\right|\right)^{p_{0}} d t \lesssim(1-r)^{\frac{1}{2}}$ for some $p_{0}>\frac{1}{2}$, then the same clearly holds for all $p \geq p_{0}$. Therefore it suffices to prove the assertion for $0<p \leq 1$. Since

$$
1-|S(z)|=1-\prod_{n=1}^{\infty}\left|S_{n}(z)\right| \leq \sum_{n=1}^{\infty}\left(1-\left|S_{n}(z)\right|\right), \quad z \in \mathbf{D},
$$

we obtain

$$
\begin{aligned}
\int_{0}^{2 \pi} & \left(1-\left|S\left(r e^{i t}\right)\right|\right)^{p} d t \leq \int_{0}^{2 \pi}\left(\sum_{n=1}^{\infty}\left(1-\left|S_{n}\left(r e^{i t}\right)\right|\right)\right)^{p} d t \\
& \leq \int_{0}^{2 \pi} \sum_{n=1}^{\infty}\left(1-\left|S_{n}\left(r e^{i t}\right)\right|\right)^{p} d t=\sum_{n=1}^{\infty} \int_{0}^{2 \pi}\left(1-\left|S_{n}\left(r e^{i t}\right)\right|\right)^{p} d t \\
& =\sum_{\gamma_{n}>\gamma_{1}(1-r)} \int_{0}^{2 \pi}\left(1-\left|S_{n}\left(r e^{i t}\right)\right|\right)^{p} d t+\sum_{\gamma_{n} \leq \gamma_{1}(1-r)} \int_{0}^{2 \pi}\left(1-\left|S_{n}\left(r e^{i t}\right)\right|\right)^{p} d t \\
& =I_{1}(r)+I_{2}(r) .
\end{aligned}
$$

By (3.3), we have

$$
I_{1}(r) \lesssim(1-r)^{\frac{1}{2}} \sum_{\gamma_{n}>\gamma_{1}(1-r)} \gamma_{n}^{\frac{1}{2}}\left(\int_{\frac{\gamma_{n}(1-r)}{2}}^{2 \gamma_{1}}+\int_{2 \gamma_{1}}^{\frac{2 \gamma_{n}}{1-r}}\right) \frac{\left(1-e^{-x}\right)^{p} d x}{x^{\frac{3}{2}}\left(2-\frac{x(1-r)}{\gamma_{n}}\right)^{\frac{1}{2}}}
$$


If $\gamma_{n} \geq 2 \gamma_{1}(1-r)$, then

$$
I_{3}(r)=\int_{\frac{\gamma_{n}(1-r)}{2}}^{2 \gamma_{1}} \frac{\left(1-e^{-x}\right)^{p} d x}{x^{\frac{3}{2}}\left(2-\frac{x(1-r)}{\gamma_{n}}\right)^{\frac{1}{2}}} \leq \int_{\frac{\gamma_{n}(1-r)}{2}}^{2 \gamma_{1}} \frac{d x}{x^{\frac{3}{2}-p}} \asymp \gamma_{n}^{\widehat{p}-\frac{1}{2}}(1-r)^{p-\frac{1}{2}} h_{p}(r), \quad n \in \mathbf{N},
$$

because $1-e^{-s} \leq s$ for $s \in(0, \infty)$. If $\gamma_{1}(1-r)<\gamma_{n}<2 \gamma_{1}(1-r)$, then

$$
\begin{aligned}
I_{3}(r) & =\left(\frac{1-r}{\gamma_{n}}\right)^{\frac{1}{2}} \int_{\frac{(1-r)^{2}}{2}}^{\frac{2 \gamma_{1}(1-r)}{\gamma_{n}}} \frac{\left.\left(1-\exp \left(-\frac{\gamma_{n} y}{1-r}\right)\right)\right)^{p} d y}{y^{\frac{3}{2}}(2-y)^{\frac{1}{2}}} \\
& \leq\left(\frac{1-r}{\gamma_{n}}\right)^{\frac{1}{2}}\left(\int_{\frac{(1-r)^{2}}{2}}^{1}+\int_{1}^{2}\right) \frac{\left.\left(1-\exp \left(-\frac{\gamma_{n} y}{1-r}\right)\right)\right)^{p} d y}{y^{\frac{3}{2}}(2-y)^{\frac{1}{2}}} \\
& \lesssim\left(\frac{1-r}{\gamma_{n}}\right)^{\frac{1}{2}-p} \int_{\frac{(1-r)^{2}}{2}}^{1} \frac{d y}{y^{\frac{3}{2}-p}}+\gamma_{1}^{-\frac{1}{2}} \asymp \gamma_{n}^{\widehat{p}-\frac{1}{2}}(1-r)^{p-\frac{1}{2}} h_{p}(r), \quad n \in \mathbf{N} .
\end{aligned}
$$

Hence $I_{3}(r) \lesssim \gamma_{n}^{\widehat{p}-\frac{1}{2}}(1-r)^{p-\frac{1}{2}} h_{p}(r)$. By an analogous manner, we can also show that

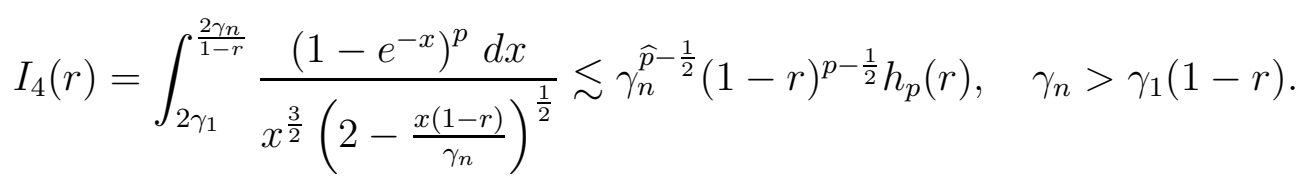

Now, by using the estimates for $I_{3}$ and $I_{4}$ in (3.4), we obtain $I_{1}(r) \lesssim\|\gamma\|_{\ell^{\widehat{p}}}^{\widehat{p}}(1-$ $r)^{p} h_{p}(r)$. Further, since

$$
\begin{aligned}
\int_{0}^{2 \pi}\left(1-\left|S_{n}\left(r e^{i t}\right)\right|\right)^{p} d t & =\int_{0}^{2 \pi}\left(1-\exp \left(-\gamma_{n} \frac{1-r^{2}}{\left|\xi_{n}-r e^{i t}\right|^{2}}\right)\right)^{p} d t \\
& \lesssim \gamma_{n}^{p}(1-r)^{p} \int_{0}^{2 \pi} \frac{d t}{\left|1-r e^{i t}\right|^{2 p}} \lesssim \gamma_{n}^{p}(1-r)^{\frac{1}{2}} h_{p}(r), \quad n \in \mathbf{N}
\end{aligned}
$$

we have

$$
I_{2}(r) \lesssim(1-r)^{\frac{1}{2}} h_{p}(r) \sum_{\gamma_{n} \leq \gamma_{1}(1-r)} \gamma_{n}^{p} \lesssim\|\gamma\|_{\ell^{\widehat{p}}}^{\widehat{p}}(1-r)^{p} h_{p}(r) .
$$

By combining the estimates for $I_{1}$ and $I_{2}$ we deduce the assertion.

Note that Theorem 8 in the case $p=1$ has been proved earlier in [1], but the proof there is based on different methods. The following corollary shows that Theorem 8 is sharp for $p \geq \frac{1}{2}$.

Corollary 9. If $S$ is a singular inner function, then the following statements are equivalent:

(a) $S$ is a purely atomic singular inner function associated with $\gamma \in \ell^{\frac{1}{2}}$;

(b) $M_{1 / 2}^{1 / 2}\left(r, S^{\prime}\right) \lesssim \log \left(\frac{1}{1-r}\right)$, as $r \rightarrow 1^{-}$;

(c) $\int_{0}^{2 \pi}\left(1-\left|S\left(r e^{i t}\right)\right|\right)^{\frac{1}{2}} d t \lesssim(1-r)^{\frac{1}{2}} \log \left(\frac{1}{1-r}\right)$, as $r \rightarrow 1^{-}$;

(d) there exists $\frac{1}{2}<p<\infty$ such that $\int_{0}^{2 \pi}\left(1-\left|S\left(r e^{i t}\right)\right|\right)^{p} d t \lesssim(1-r)^{\frac{1}{2}}$, as $r \rightarrow 1^{-}$;

(e) $\int_{0}^{2 \pi}\left(1-\left|S\left(r e^{i t}\right)\right|\right)^{p} d t \lesssim(1-r)^{\frac{1}{2}}$, as $r \rightarrow 1^{-}$, for each $\frac{1}{2}<p<\infty$.

Proof. The statements (a) and (b) are equivalent by [5, Theorem 2.2]. Moreover, (a) implies (c)-(e) by Theorem 8, and (c) implies (b) by the Schwarz-Pick lemma. 
To complete the proof, it suffices to show that (d) implies (a). If (a) does not hold, then the proof of [5, Theorem 2.2] yields

$$
(1-r)^{-\frac{1}{2}} \int_{0}^{2 \pi}\left(1-\left|S\left(r e^{i t}\right)\right|\right)^{p} d t \rightarrow \infty, \quad r \rightarrow 1^{-},
$$

for each $0<p<\infty$, and this clearly contradicts (d). Thus the assertion is proved.

If an inner function $\Theta$ satisfies either

$$
\left(\log \frac{1}{1-r}\right)^{-1} M_{1 / 2}^{1 / 2}\left(r, \Theta^{\prime}\right) \longrightarrow 0^{+}, \quad r \rightarrow 1^{-}
$$

or

$$
\frac{\int_{0}^{2 \pi}\left(1-\left|\Theta\left(r e^{i t}\right)\right|\right)^{p} d t}{(1-r)^{\frac{1}{2}}} \longrightarrow 0^{+}, \quad p>\frac{1}{2}, \quad r \rightarrow 1^{-},
$$

then it is a Blaschke product. In the first case, the assertion is a direct consequence of [5, Theorem 2.1]. In the latter case, the assertion follows by (1.6) and a special case of the Beurling factorization according to which every non-constant inner function is either a Blaschke product, a singular inner function or a product of the previous ones [10].

Theorem 3 for $\omega \in \widehat{\mathcal{D}}_{p}$ can be proved by using Theorem 8 and (1.1), as will be shown later. To deal with the remaining case, we will use the following result which shows that $M_{p}^{p}\left(r, S^{\prime}\right) \asymp h_{p}(r)$ for each purely atomic singular inner function $S$ associated with a measure having a separate mass point.

Theorem 10. Let $0<p<\infty$ and $\widehat{p}=\min \left\{\frac{1}{2}, p\right\}$. Let $S$ be the purely atomic singular inner function associated with $\left\{\xi_{n}\right\}$ and $\gamma=\left\{\gamma_{n}\right\} \in \ell^{\widehat{p}}$, and having a separate mass point in its inducing measure. Then there exists $r_{0}=r_{0}(p, S) \in(0,1)$ such that

$$
\frac{\int_{0}^{2 \pi}\left(1-\left|S\left(r e^{i t}\right)\right|\right)^{p} d t}{(1-r)^{p}} \asymp M_{p}^{p}\left(r, S^{\prime}\right) \asymp h_{p}(r), \quad r_{0}<r<1,
$$

where $h_{p}$ is as in Theorem 8 .

Proof. Let $\xi_{j}$ be a separate mass point and write $S=\prod_{n=1}^{\infty} S_{n}$, where $S_{n}=S_{\gamma_{n}, \xi_{n}}$. It suffices to show that $M_{p}^{p}\left(r, S^{\prime}\right) \gtrsim M_{p}^{p}\left(r, S_{j}^{\prime}\right)$ for $r$ close enough to one because then this together with Theorem 8, the Schwarz-Pick lemma and the main result of [26] yields

$$
h_{p}(r) \asymp \frac{\int_{0}^{2 \pi}\left(1-\left|S\left(r e^{i t}\right)\right|\right)^{p} d t}{(1-r)^{p}} \gtrsim M_{p}^{p}\left(r, S^{\prime}\right) \gtrsim M_{p}^{p}\left(r, S_{j}^{\prime}\right) \asymp h_{p}(r), \quad r_{0}<r<1 .
$$

To prove $M_{p}^{p}\left(r, S^{\prime}\right) \gtrsim M_{p}^{p}\left(r, S_{j}^{\prime}\right)$, note first that

$$
\begin{aligned}
\left|S^{\prime}(z)\right| & =\left|-2 \sum_{n=1}^{\infty} \frac{\gamma_{n} \xi_{n}}{\left(z-\xi_{n}\right)^{2}} \exp \left(\sum_{k=1}^{\infty} \gamma_{k} \frac{z+\xi_{k}}{z-\xi_{k}}\right)\right| \\
& =2\left|\sum_{n=1}^{\infty} \frac{\gamma_{n} \xi_{n}}{\left(z-\xi_{n}\right)^{2}}\right| \exp \left(-\sum_{k=1}^{\infty} \gamma_{k} \frac{1-|z|^{2}}{\left|z-\xi_{k}\right|^{2}}\right) \\
& \geq\left(\frac{\gamma_{j}}{\left|z-\xi_{j}\right|^{2}}-\sum_{n \neq j} \frac{\gamma_{n}}{\left|z-\xi_{n}\right|^{2}}\right) \exp \left(-\sum_{k=1}^{\infty} \gamma_{k} \frac{1-|z|^{2}}{\left|z-\xi_{k}\right|^{2}}\right) .
\end{aligned}
$$


For each $k \in \mathbf{N}$, write $\xi_{k}=e^{i \theta_{k}}$, where $0 \leq \theta_{k}<2 \pi$. By the hypothesis, there exists $\varepsilon=\varepsilon(S) \in(0, \pi)$ such that $\left|\theta_{j}-\theta_{k}\right|>\varepsilon$ for all $k \neq j$. Let first $\left|t-\theta_{j}\right|<\frac{\varepsilon}{2}$ and $r>\frac{1}{2}$. Then (3.2) yields

$$
\begin{aligned}
& \exp \left(-\sum_{k \neq j} \gamma_{k} \frac{1-r^{2}}{\mid r e^{i t}-e^{\left.i \theta_{k}\right|^{2}}}\right)=\exp \left(-\sum_{k \neq j} \gamma_{k} \frac{1-r^{2}}{(1-r)^{2}+r \mid 1-e^{\left.i\left(t-\theta_{k}\right)\right|^{2}}}\right) \\
& \quad \geq \exp \left(-6 \sum_{k \neq j} \frac{\gamma_{k}}{r\left(t-\theta_{k}\right)^{2}}\right) \geq \exp \left(-\frac{48}{\varepsilon^{2}} \sum_{k \neq j} \gamma_{k}\right) \geq \exp \left(-\frac{48}{\varepsilon^{2}}\|\gamma\|_{\ell^{1}}\right)
\end{aligned}
$$

and

$$
f\left(r e^{i t}\right)=\frac{\gamma_{j}}{\left|r e^{i t}-e^{i \theta_{j}}\right|^{2}}-\sum_{n \neq j} \frac{\gamma_{n}}{\left|r e^{i t}-e^{i \theta_{n}}\right|^{2}} \geq \frac{\gamma_{j}}{(1-r)^{2}+r\left(t-\theta_{j}\right)^{2}}-\frac{24}{\varepsilon^{2}}\|\gamma\|_{\ell^{1}} .
$$

Set $M=M(S)=\left(24 / \varepsilon^{2}\right)\|\gamma\|_{\ell^{1}}$. Then, for $r>\max \left\{\frac{1}{2}, 1-\sqrt{\frac{\gamma_{j}}{4 M}}\right\}$ and $\left|t-\theta_{j}\right|<$ $\sqrt{\frac{\gamma_{j}}{4 M}}$, we obtain

$$
f\left(r e^{i t}\right) \geq \frac{1}{2} \frac{\gamma_{j}}{(1-r)^{2}+r\left(t-\theta_{j}\right)^{2}} \geq \frac{\gamma_{j}}{6 \mid r e^{i t}-e^{\left.i \theta_{j}\right|^{2}}} .
$$

If $\alpha=\min \left\{\sqrt{\frac{\gamma_{j}}{4 M}}, \frac{\varepsilon}{2}\right\}$, then, by combining the estimates above, we deduce

$$
M_{p}^{p}\left(r, S^{\prime}\right) \geq \int_{\theta_{j}-\alpha}^{\theta_{j}+\alpha}\left|S^{\prime}\left(r e^{i t}\right)\right|^{p} d t \gtrsim \int_{\theta_{j}-\alpha}^{\theta_{j}+\alpha}\left|S_{\gamma_{j}, \xi_{j}}^{\prime}\left(r e^{i t}\right)\right|^{p} d t \asymp M_{p}^{p}\left(r, S_{\gamma_{j}, \xi_{j}}^{\prime}\right)
$$

for $r$ close enough to one depending on $p$ and $S$. Thus the assertion is proved.

With these results in hand we can easily establish Theorem 3.

Proof of Theorem 3. Let us begin with the case $\omega \in \widehat{\mathcal{D}}_{p}$. By multiplying (3.1) by $r \omega(r)$ and integrating with respect to $r$ we obtain

$$
\int_{\mathbf{D} \backslash D\left(0, \frac{1}{2}\right)}\left(\frac{1-|S(z)|^{2}}{1-|z|^{2}}\right)^{p} \omega(z) d A(z) \asymp \int_{\frac{1}{2}}^{1} h_{p}(r) \omega(r) r d r,
$$

where $S$ and $h_{p}$ are as in Theorem 8 . The assertion in Theorem 3 for $\omega \in \widehat{\mathcal{D}}_{p}$ now follows by (1.1). If $S$ is associated with a measure having a separate mass point, then the assertion can be proved by an analogous manner using (3.5).

The statement in Theorem 3 for $p \leq \frac{1}{2}$ is actually valid for each radial weight $\omega$ and each purely atomic singular inner function $S$ with $\gamma \in \ell^{\widehat{p}}$. This is an immediate consequence of Theorem 8, the Schwarz-Pick lemma and [5, Theorem 2.2]. Further, it is worth observing that $S^{\prime} \in H^{p}$ if and only if $p<\frac{1}{2}$.

Acknowledgements. This paper is a part of the third author's PhD thesis. The authors thank the thesis reviewers Igor Chyzhykov and Toshiyuki Sugawa, and the opponent José Ángel Peláez for careful reading of the manuscript and valuable comments. 


\section{References}

[1] Ahern, P.: The mean modulus and the derivative of an inner function. - Indiana Univ. Math. J. 28:2, 1979, 311-347.

[2] Ahern, P.: The Poisson integral of a singular measure. - Canad. J. Math. 35:4, 1983, 735-749.

[3] Ahern, P. R., and D. N. Clark: On inner functions with $B^{p}$ derivative. - Michigan Math. J. 23:2, 1976, 107-118.

[4] Ahern, P.R., and D. N. Clark: On inner functions with $H^{p}$ derivative. - Michigan Math. J. 21, 1974, 115-127.

[5] Ahern, P., and M. Jevtić: Mean modulus and the fractional derivative of an inner function. - Complex Variables Theory Appl. 3:4, 1984, 431-445.

[6] Aleman, A., and D. Vukotić: On Blaschke products with derivatives in Bergman spaces with normal weights. - J. Math. Anal. Appl. 361:2, 2010, 492-505.

[7] Conn, W. S.: On the $H^{p}$ classes of derivatives of functions orthogonal to invariant subspaces. - Michigan Math. J. 30:2, 1983, 221-229.

[8] Cohn, W. S.: Radial limits and star invariant subspaces of bounded mean oscillation. - Amer. J. Math. 108:3, 1986, 719-749.

[9] Colwell, P.: Blaschke products: Bounded analytic functions. - University of Michigan Press, Ann Arbor/Michigan, 1985.

[10] Duren, P.: Theory of $H^{p}$ spaces. - Academic Press, New York-London, 1970.

[11] Frichin, E., and J. Mashreghi: Integral means of the derivatives of Blaschke products. Glasg. Math. J. 50:2, 2008, 233-249.

[12] Garnett, J.: Bounded analytic functions. Revised 1st edition. - Springer, New York, 2007.

[13] Girela, D., C. González, and M. Jevtić: Inner functions in Lipschitz, Besov, and Sobolev spaces. - Abstr. Appl. Anal. 2011, Art. ID 626254, 1-26.

[14] Girela, D., and J. A. Peláez: On the derivative of infinite Blaschke products. - Illinois J. Math. 48:1, 2004, 121-130.

[15] Girela, D., and J. A. PelÁez: On the membership in Bergman spaces of the derivative of a Blaschke product with zeros in a Stolz domain. - Canad. Math. Bull. 49:3, 2006, 381-388.

[16] Girela, D., J.A. Peláez, and D. Vukotić: Integrability of the derivative of a Blaschke product. - Proc. Edinb. Math. Soc. (2) 50:3, 2007, 673-687.

[17] Girela, D., J. A. Peláez, and D. Vukotić: Uniformly discrete sequences in regions with tangential approach to the unit circle. - Complex Var. Elliptic Equ. 52:2-3, 2007, 161-173.

[18] Gluchoff, A.: The mean modulus of a Blaschke product with zeroes in a nontangential region. - Complex Variables Theory Appl. 1:4, 1983, 311-326.

[19] Gluchoff, A.: On inner functions with derivative in Bergman spaces. - Illinois J. Math. 31:3, $1987,518-528$.

[20] Gröhn, J., and A. Nicolau: Inner functions in certain Hardy-Sobolev spaces. - J. Funct. Anal. 272:6, 2017, 2463-2486.

[21] Kim, H.-O.: Derivatives of Blaschke products. - Pacific J. Math. 114:1, 1984, 175-190.

[22] KutBi, M. A.: Integral means for the first derivative of Blaschke products. - Kodai Math. J. $24: 1,2001,86-97$.

[23] McDonald, G., and C. Sundberg: Toeplitz operators on the disc. - Indiana Univ. Math. J. 28:4, 1979, 595-611.

[24] McKenna, P.: Discrete Carleson measures and interpolation problems. - Michigan Math. J. $24: 3,1977,311-319$.

[25] Mashreghi, J.: Derivatives of inner functions. - Fields Inst. Monogr. 31, Springer, New York; Fields Institute for Research in Mathematical Sciences, Toronto, ON, 2013. 
[26] Mateljević, M., and M. Pavlović: On the integral means of derivatives of the atomic function. - Proc. Amer. Math. Soc. 86:3, 1982, 455-458.

[27] Pavlović, M.: Introduction to function spaces on the disk. - Posebna Izdanja 20, Matematički Institut SANU, Belgrade, 2004.

[28] PelÁez, J.A., and J. RÄTtyä: Weighted Bergman spaces induced by rapidly increasing weights. - Mem. Amer. Math. Soc. 227:1066, 2014.

[29] PelÁez, J. A., and J. RÄTtYä: Embedding theorems for Bergman spaces via harmonic analysis. - Math. Ann. 362:1-2, 2015, 205-239.

[30] PelÁez, J. A., and J. RÄтtyä: Weighted Bergman projection in $L^{\infty}$. - Preprint.

[31] PÉrez-GonzÁlez, F., and J. RÄTtyä: Derivatives of inner functions in weighted Bergman spaces and the Schwarz-Pick lemma. - Proc. Amer. Math. Soc. 145:5, 2017, 2155-2166.

[32] Protas, D.: Blaschke products with derivative in function spaces. - Kodai Math. J. 34:1, 2011, 124-131.

[33] Protas, D.: Blaschke products with derivative in $H^{p}$ and $B^{p}$. - Michigan Math. J. 20, 1973, 393-396.

[34] Protas, D.: Mean growth of the derivative of a Blaschke product. - Kodai Math. J. 27:3, 2004, 354-359.

[35] Shields, A. L., and D. L. Williams: Bounded projections, duality, and multipliers in spaces of analytic functions. - Trans. Amer. Math. Soc. 162, 1971, 287-302.

Received 12 August 2016 • Received 15 December 2016 • Accepted 23 December 2016 